\title{
Aligning Parallel Arrays to Reduce Communication
}

\author{
Thomas J. Sheffler \\ Robert Schreiber \\ John R. Gilbert \\ Siddhartha Chatterjee
}

The Research Institute for Advanced Computer Science is operated by Universities Space Research Association, The American City Building, Suite 212, Columbia, MD 21044, (410) 730-2656

Work reported herein was supported by NASA via Contract NAS 2-13721 between NASA and the Universities Space Research Association (USRA). Work was performed at the Research Institute for Advanced Computer Science (RIACS), NASA Ames Research Center, Moffett Field, CA 94035-1000. 



\title{
Aligning Parallel Arrays to Reduce Communication
}

\author{
Thomas J. Sheffler * Robert Schreiber * John R. Gilbert ${ }^{\dagger} \quad$ Siddhartha Chatterjee *
}

\begin{abstract}
Axis and stride alignment is an important optimization in compiling data-parallel programs for distributed-memory machines. We previously developed an optimal algorithm for aligning array expressions. Here, we examine alignment for more general program graphs. We show that optimal alignment is NP-complete in this setting, so we study heuristic methods.

This paper makes two contributions. First, we show how local graph transformations can reduce the size of the problem significantly without changing the best solution. This allows more complex and effective heuristics to be used. Second, we give a heuristic that can explore the space of possible solutions in a number of ways. We show that some of these strategies can give better solutions than a simple greedy approach proposed earlier. Our algorithms have been implemented; we present experimental results showing their effect on the performance of some example programs running on the CM-5.
\end{abstract}

\section{Introduction}

Placing arrays to enhance data locality is an important problem in implementing array-parallel languages on distributed-memory parallel computers. Languages such as High Performance Fortran [7] require the user to provide data placement directives in the source code. There has also been considerable interest in automating the task of data placement $[1,3,4,8,9,10,12]$. This compiler optimization is important for ensuring the portability of new scientific codes and for supporting old codes developed without a distributed memory model in mind.

Data placement optimization may be described as a two-step process. First, the alignment phase examines the relationships between array objects in a program and determines the manner in which corresponding array elements should be co-located to reduce communication costs. Second, the distribution phase partitions arrays over processor memories. The alignment phase deals with the relative positions of array objects in an architecture-independent framework, while distribution considers their absolute positions in a distributed memory.

This paper considers the following alignment problem: Given a data-parallel program and a $t$ dimensional index space called the template, find a mapping of each array object to the template so as to minimize communication costs. The mapping of an array is called its position with respect to the template space. It is made up of three components: axis, stride and offset. The axis alignment of an array determines the correspondence between array axes and template axes, the stride component gives the spacing

\footnotetext{
-Research Institute for Advanced Computer Science, Mail Stop T27A-1, NASA Ames Research Center, Moffett Field, CA 94035-1000 (sheffler@riacs.edu, schreibr@riacs.edu, sc@riacs.edu). The work of these authors was supported by the NAS Systems Division via Contract NAS 2-13721 between NASA and the Universities Space Research Association (USRA).

${ }^{\dagger}$ Xerox Palo Alto Research Center, 3333 Coyote Hill Road, Palo Alto, CA 94304-1314 (gilbert@parc.xerox.com). Copyright (C) 1993, 1994 by Xerox Corporation. All rights reserved. Phone: 415-812-4487. Fax: 415-812-4471.
} 
with which each array axis is mapped to a template axis, and the offset gives the displacement of the origin of the array object from the template's origin. Axis and stride play the biggest role in reducing communication costs because correcting axis and stride misalignment requires general all-to-all communication. This paper addresses the axis/stride alignment problem.

\subsection{Related Work}

Knobe, Lukas and Steele [9] laid a foundation for data layout optimization of parallel programs. They addressed axis, stride and offset alignment in a unified framework. This paper amplifies their claims of the importance of data layout optimization, and improves upon their methods in several ways.

First, we use a more comprehensive cost model. This is inherited directly from our alignment-distribution graph representation of data-parallel programs [4]. We also defer offset concerns to a later phase of alignment, because the shift communication needed to change offset is typically much less expensive than the general communication needed to change axis or stride.

Second, we develop a heuristic optimization framework that is more flexible than the strictly greedy algorithm of Knobe, Lukas and Steele. Our experimental results confirm that the greedy heuristic can miss solutions that our algorithm finds.

Third, we show how to use local graph transformations to reduce the size of the optimization problem without changing its best solution. This reduction allows us to use more complex and effective heuristics than would be feasible for the unreduced graph.

In other related work, $\mathrm{Li}$ and Chen [10] addressed axis alignment alone, using a representation called a component affinity graph. Edges of this graph represent axis constraints to be satisfied. Their optimization algorithm is also greedy, but it is their cost model that most differentiates their work from ours. They formulate the problem as a graph with large and small weight edges, such that large edges are infinitely heavier than small edges. The optimization procedure finds a maximal weight set of edges that satisfy the constraints. Our cost model reflects the actual communication cost of a parallel program more accurately.

Anderson and Lam [1] addressed alignment in a linear algebraic framework. They permit a broader class of alignments than we do, but often sacrifice parallelism to reduce communication. The tradeoff between communication and parallelism is intimately related to parameters of the target machine. Our approach discovers alignment constraints that depend only on the source program, providing information that is useful on any target machine. As a result, we retain as much parallelism as is present in the source code.

Earlier, we developed an exact algorithm called compact dynamic programming for finding minimum cost alignments of tree-structured computations (namely, expressions). We suggested using that algorithm as a heuristic for arbitrary programs, but experiments showed that it often makes poor alignment decisions because it uses only local information. Our new algorithm makes better use of global connectivity information.

\subsection{Organization}

This paper begins by reviewing the alignment-distribution graph as a means for representing data-parallel programs with alignment information made explicit. From there, we show how to construct another graph, called the constraint graph, on which the optimization algorithm is performed. Our heuristic optimization algorithm finds a maximal set of edges of the constraint graph that may be satisfied, leaving other edges to carry realignment communication costs. An important part of this framework is the development of a new linear time algorithm that verifies the existence of a communication-free labeling for a given subgraph. We also show that the problem of minimizing the number of template dimensions required by such a subgraph 


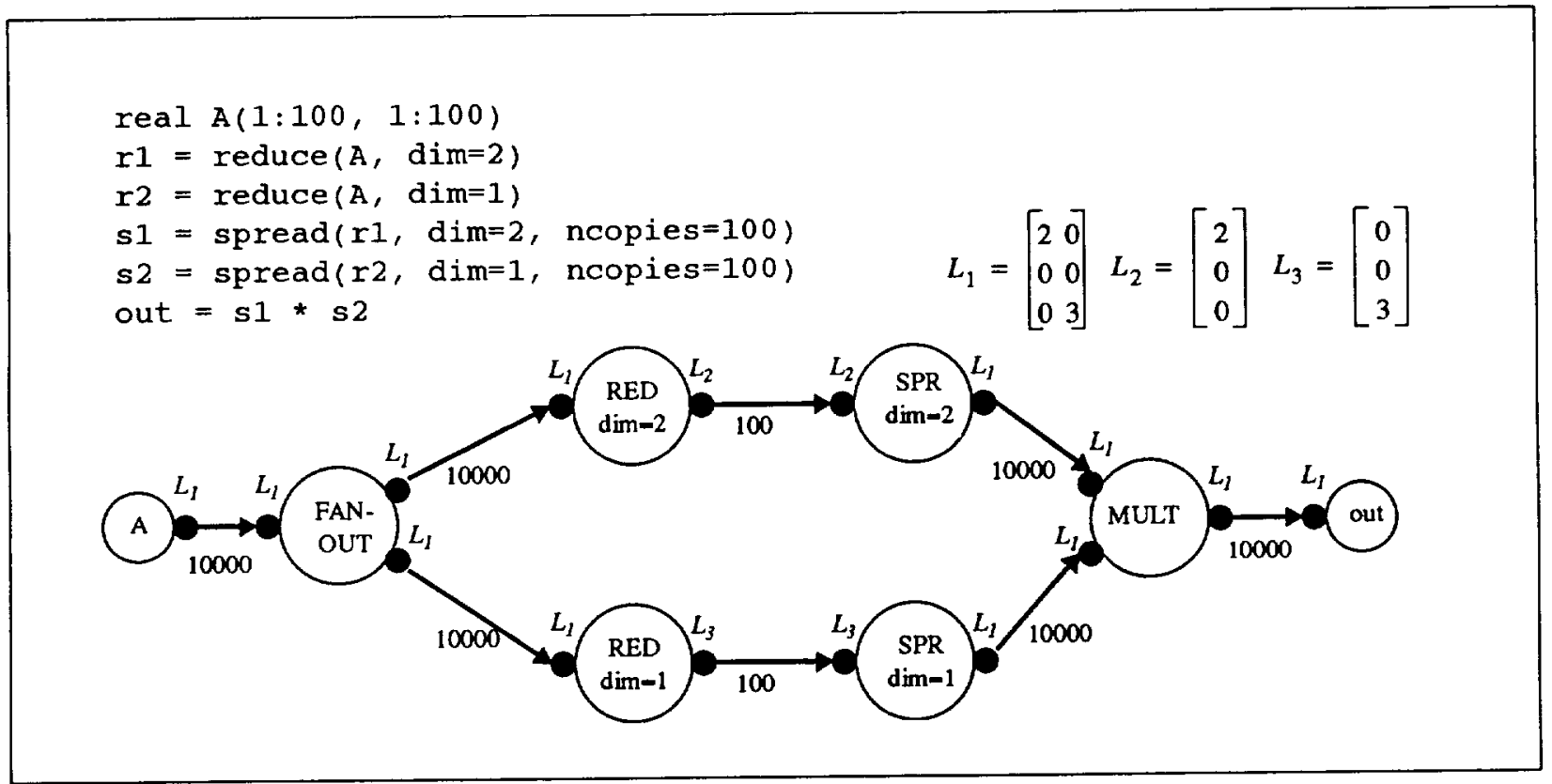

Figure 1: A code fragment using reduce and spread and its ADG. Data weights on edges represent the cost of communication. Each port has a position label. The ADG represents data flow in a program. It can also include nodes that reflect control flow due to branches or loops.

is NP-complete. Concluding sections compare this approach to others and summarize test results conducted with an implementation of the algorithm described.

\section{Representing the Alignment Problem}

Previously, we developed a representation of data-parallel programs called the alignment-distribution graph (ADG) [4] to evaluate data layout decisions made during compilation. The ADG is based on static single assignment form [5], but incorporates a "position semantics" that makes each communication operation of the program explicit. This section shows how to distill the ADG to a simpler graph that only represents axis and stride.

\subsection{The Alignment-Distribution Graph}

An example ADG for a Fortran 90 code fragment appears in Figure 1. Nodes in the graph represent computation, and edges represent flow of data. An endpoint of an edge is called a port and represents an array object with a specified position. Thus, an edge transforms an array object from one position to another. Realignment occurs whenever the two ports of an edge have different positions. A node constrains the relative positions of its ports, which are the locations of its operands and results. For example, the elementwise MULTIPLY node in the figure requires that its arguments and result ports be aligned identically. In the figure, positions are represented as the matrices, $L_{1}, L_{2}$ and $L_{3}$. These are described next.

A position is an affine mapping from the coordinates of an array object of $d$ dimensions to the coordinates of the template of $t$ dimensions. An array point $p_{d} \in \mathbf{Z}^{d}$ is mapped to a template location $p_{t} \in \mathbf{Z}^{t}$ by the 
following matrix equation:

$$
p_{t}=L p_{d}+f
$$

where $L$ is an $t \times d$ matrix of integers and $f$ is a column vector specifying the offset component of the mapping. Here, we consider only axis and stride alignment, so the offset component becomes zero. Thus, a position is completely determined by the array $L$. For example, the two-dimensional object $A$ with position

$$
L_{1}=\left[\begin{array}{ll}
2 & 0 \\
0 & 0 \\
0 & 3
\end{array}\right]
$$

is mapped to a three-dimensional template with its first axis mapped to the first axis of the template with stride 2 , and its second axis mapped onto the third axis of the template with stride 3.

Suppose axis $j$ of an array object is mapped to axis $i$ of the template with stride $s$. Then column $j$ of the object's position matrix has exactly one nonzero element, in row $i$, with value $s$. Row $i$ has no other nonzeros, because at most one array axis maps to each template axis. We call a matrix with exactly one nonzero in each column and at most one nonzero in each row a $D$-matrix.

The data weight of the edge is the total amount of data it transfers. If the endpoints of an edge have different positions, the edge incurs communication cost equal to its data weight. (Our experiments in Section 5 indicate that this "discrete metric" is a good model of axis/stride realignment cost; the ADG framework can support other cost metrics as well [4].)

The total cost of an assignment of positions to ADG ports is the sum of the data weights on edges whose endpoints are at different positions. In ADG terms, the objective of alignment is to find an assignment of positions that minimizes this cost.

\subsection{The Constraint Graph}

The ADG represents alignment and distribution for arrays in a parallel program. In this section, we transform the ADG into a simpler graph, called the constraint graph (CG), that is specific to alignment analysis. The CG unifies the representations of positions and constraints, and efficiently captures the costs associated with each constraint. Each port in the ADG becomes a vertex in the CG. Edges and nodes in the ADG contribute constraints, which are represented as edges in the CG. Figure 2 illustrates the transformation of a line of source code into an ADG node and then into a CG, with positions and constraints given as matrices. The rest of this section describes the construction and use of the CG.

An ADG node imposes a constraint on the positions of its ports that cannot be violated. A constraint is a mapping from the coordinate space of one array object to another. If a node involves two array objects, $x$ and $y$, and imposes a constraint on the position of $y$ with respect to $x$, then this constraint can be written as

$$
L_{y}=L_{x} C_{x y},
$$

where $C_{x y}$ is a constraint matrix. A constraint matrix is a D-matrix having at least as many rows as columns.

Because both $L_{x}$ and $L_{y}$ describe mappings to the template, this equation says that corresponding elements from the two array objects are mapped to the same template site. For a node involving three or more ports, one is designated the reference port, and the constraints are expressed relative to it. The construction of constraint matrices for the various node types of the ADG is straightforward [4].

An ADG edge between two ports, $x$ and $y$, imposes an equality constraint that may be violated at a specified cost, $W_{x y}$. We write such as constraint in the same form

$$
L_{y}=L_{x} C_{x y}
$$



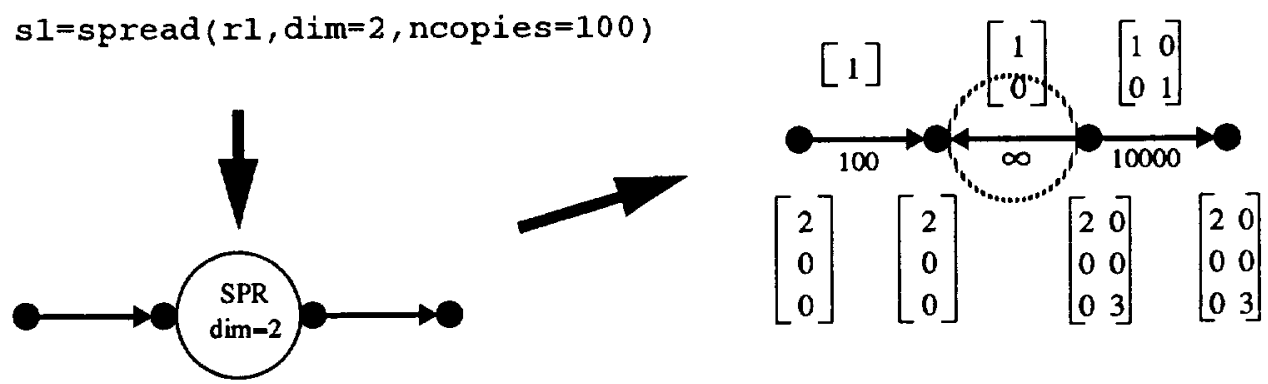

Figure 2: A code fragment, its translation into an ADG node, and the resulting constraint graph. Each port has a position (shown below) and each edge imposes a constraint (shown above). A position labeling satisfies an edge if the head position is the product of the tail position and edge constraint.

where in this case $C_{x y}$ is an identity matrix. Positions matrices are D-matrices, and it turns out that constraint matrices are also D-matrices.

The CG is constructed from these constraints. For each array object $x$, (that is, each ADG port), there is a vertex $v_{x}$. For each constraint $L_{y}=L_{x} C_{x y}$, there is a directed edge from $v_{x}$ to $v_{y}$ with label $C_{x y}$. Each edge also has an associated weight $W_{x y}$, which is the communication cost of moving an object from position $L_{x}$ to $L_{y}$ if the constraint is not satisfied. An edge in the CG that corresponds to a node constraint in the ADG has $W_{x y}=\infty$.

A labeling of the CG is called "communication-free" if it satisfies every edge constraint, and a CG is called "satisfiable" if at least one such labeling exists.

This simple formulation captures all of the possible constraints pertinent to alignment analysis among array objects in High Performance Fortran. For example, constraint matrices can express relations between arrays that are projections, reductions or sections of one another. The CG may be simplified even further. Section 4 discusses graph contraction operations that often reduce an alignment problem to a graph of only a few vertices.

\section{An Axis/Stride Labeling Algorithm}

The alignment problem is surprisingly hard. It is NP-complete even when restricted to only axis alignment for two-dimensional arrays in a two-dimensional template. Thus, we must be satisfied with heuristic or approximate solutions.

Theorem 1 Min-cost labeling of an ADG is NP-complete, even considering only straight-line programs involving two-dimensional arrays with the transpose and addition operations.

Proof: It is easy to see that min-cost labeling is in NP since the cost of a given axis labeling can be computed in polynomial time. We proceed by reduction from "Bipartite Subgraph (GT25)", [6] which is the following problem: Given a graph $G$ and an integer $k$, is there a bipartite subgraph of $G$ with at least $k$ edges? (Equivalently, is there a way to 2 -color the nodes of $G$ that violates the color condition for at most $t=e-k$ edges?) This is NP-complete even if $\mathrm{G}$ has only vertices of degree 2 and 3. 
We first transform $G$ to a graph with two kinds of edges, "opposite" edges whose endpoints are to be colored differently and "same" edges whose endpoints are to be colored the same. Each original edge of $G$ is an "opposite" edge. Split each degree-2 node of $\mathrm{G}$ into two nodes joined by a new "same" edge. Split each degree-3 node of $G$ into a 4-vertex star, each of whose leaves is incident on an original edge, with 3 new "same" edges. It is easy to see that $G$ can be 2 -colored in a way that violates at most $t$ edges if and only if the transformed graph can be. Furthermore, since each "opposite" edge shares a degree-2 endpoint with a "same" edge, the transformed graph has an optimal 2-coloring that violates only "same" edges.

Now each degree-2 vertex is incident on one "opposite" edge and one "same" edge, and each degree-3 vertex is incident on three "same" edges. Construct an ADG by replacing each "opposite" edge and its two endpoint vertices with a transpose node, and replacing each degree- 3 vertex with an addition node. (Formally, replace each degree- 3 vertex with a node, then direct the edges so no degree- 3 node is a source or a sink, then make the ones with in-degree 2 addition nodes.) Each edge of the ADG is a "same" edge of the transformed graph. Each "opposite" edge of the transformed graph (i.e. each edge of the original graph) is a transpose node of the ADG. A min-cost labeling of the ADG corresponds to an optimal 2-coloring of the transformed graph that violates only "same" edges.

\subsection{Outline of the Algorithm}

Let $G$ be a given CG. Like the greedy algorithms of Knobe, Lukas and Steele [9] and Li and Chen [10], our algorithm finds a maximal satisfiable subgraph of $G$. Our algorithm is not strictly greedy-it can discard edges as well as add them, and therefore will ordinarily explore a larger set of feasible solutions.

Our algorithm builds a maximal satisfiable subgraph $G^{\prime}$. Initially $G^{\prime}$ contains all of the vertices of $G$, but all edges are excluded. At each step, an excluded edge is conditionally added to $G^{\prime}$ and a subroutine is-satisfiable determines if there exists a communication-free labeling for the augmented graph $G^{\prime}$.

The optimization algorithm proceeds as follows:

1. Include an excluded edge.

2. If the resulting graph is satisfiable (see Section 3.2) then accept the new edge and go to Step 1.

Else, find a minimum-weight cut set $E$ in $G^{\prime}$ of edges between the endpoints of the edge $e$. The graph including $e$ but with $E$ removed is guaranteed to be satisfiable (see below). However, there may be edges in $E$ whose inclusion does not prohibit satisfiability. Try including each edge in $E$ back into the graph in turn, and retain in $E$ only those edges that prohibit satisfiability. $E$ is now a minimal set of edges whose removal allows a communication-free labeling of the graph with edge $e$.

3. If the weight of edge $e$ is bigger than the total weight of edge set $E$ then insert $e$ in the graph and move the edges in $E$ into the bag. Otherwise, reject $e$ and leave the graph as it was.

4. Repeat this procedure until no edges from the bag can be added into the graph.

5. Find a labeling that satisfies the final graph (see Section 3.3).

The procedure terminates because the weight of the current graph increases at every iteration (though its size may not).

Lemma 1 In step 2, the graph with e included and E removed is satisfiable. 

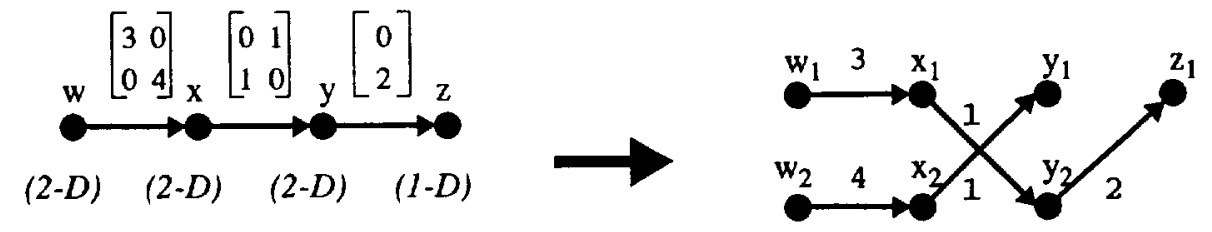

Figure 3: A new graph is constructed to verify the existence of a legal axis and stride assignment. One vertex is constructed for each axis of an array object (whose ranks are shown in italics), and edges map one axis to another with a given stride.

Proof: Each path in $G^{\prime}$ describes a constraint between its endpoints. Multiple paths between two vertices may describe incompatible constraints, in which case the graph is not satisfiable.

Prior to adding edge $e=(v, w), G^{\prime}$ is satisfiable. Thus, the constraint on edge $e$ must be incompatible with that of some other path from $v$ to $w$. Edge set $E$ is a mincut set of edges breaking all paths from $v$ to $w$. Removing $E$ leaves no constraints between $v$ and $w$ to conflict with that of edge $e$. Thus, $G^{\prime}+\{e\}-E$ is satisfiable.

\subsection{The Predicate is-satisfiable}

Given a CG, $G$, is-satisfiable determines in linear time if there is a communication-free labeling of $G$. (It does not actually have to find the labeling.) The algorithm first decides axis satisfiability, then stride satisfiability.

Axis Satisfiability: We first construct a new graph that is closely related to the component affinity graph of $\mathrm{Li}$ and Chen [10]. Each vertex of the CG is split into one vertex for each axis of its array object. Directed edges are introduced between the vertices of this new graph corresponding to the non-zero elements of the constraint matrices of the CG. This transformation is illustrated in Figure 3 for a small constraint graph fragment.

We then find the connected components of this new graph. If two vertices of the same array object are in the same connected component, then there is no communication-free solution (because the two axes would have to be mapped to the same template axis). Otherwise, there is a communication-free axis alignment that assigns each connected component to a different template axis. (This may use more template axes than necessary-see Section 3.3.)

Stride Satisfiability: For each connected component of the graph, the algorithm determines whether there is a labeling that satisfies the stride constraints by the following steps. An edge in this graph is satisfied if the product of its tail and stride labels equals its head label.

1. Find any spanning tree of the connected component. 
2. Label an arbitrary vertex " 1 " and label the rest of the vertices by multiplying (or dividing) by the stride label of the edges.

3. For each non-tree edge, check whether the stride transformation it describes is satisfied by its endpoints.

The running times of both the axis and stride phases of this algorithm are linear in the number of edges in the graph. Connected components can be found in linear time by a depth first search. Checking the vertices corresponding to each array object to determine if any are in the same component is trivial. The stride constraints can be verified during the depth-first search: as each node is visited, propagate the appropriate stride value to each non-visited neighbor. For all other neighbors, verify that the constraint on the edge is satisfied.

This algorithm performs operations similar to the conformance checking procedure of Knobe, Lukas and Steele [9], but is much simpler. They find axis conflicts by an incremental approach based on merging sets and their stride satisfiability test involves complicated array allocation functions. These differences stem from the differences in the two optimization frameworks. Our approach can generate large-scale changes to the constraint graph, requiring that we recompute satisfiability anew each time. For this reason, we developed the efficient linear-time algorithm given here. In contrast, they considered the addition of only a single edge at each step, and incremental techniques were more appropriate.

\subsection{Providing a Labeling}

The is-satisfiable procedure implicitly finds an axis and stride labeling, but its axis labeling may use more template axes than necessary. When the final maximal satisfiable subgraph is found, we label the axes by a coloring procedure as follows.

We construct another graph to describe the coloring problem: the axis quotient graph. This graph has one vertex for each connected component of the axis/stride satisfiability graph, and an undirected edge between vertices representing two connected components that occur in the same array object. A $k$-coloring of this graph corresponds to an assignment of the axes of each array object to $k$ template axes. Each color corresponds to an axis of the template, and a $k$-coloring of the graph assigns the axes of each array object to different axes of the template. Finding an optimal coloring is hard:

Theorem 2 Given an ADG that admits a communication-free labeling, axis assignment to minimize the number of template dimensions is NP-complete.

Proof: It is easy to see that this problem is in NP since we can use procedure is-satisfiable to verify the validity of a given axis alignment in polynomial time. We proceed by reduction from "Graph $k$ colorability (GT4)" [6] which is the following: Given a graph $G=(V, E)$ and a positive integer $k \leq|V|$, is $G k$-colorable, i.e., does there exist a function $f: V \rightarrow\{1,2, \ldots, k\}$ such that $f(u) \neq f(v)$ whenever $\{u, v\} \in E$ ? This is solvable in polynomial time for $k=2$, but remains NP-complete for all fixed $k \geq 3$.

Given an instance of GT4, we reduce it to an instance of our problem as follows.

- For each vertex $v_{i}$ of $G$, construct an input node in the ADG for a variable called $t_{i}$ of dimension $(1: N)$.

- For each edge $e_{m}$, construct an input node in the ADG for a two-dimensional array called $A_{m}$ with dimensions $(1: \mathrm{N}, 1: \mathrm{N})$. 
- For each edge $e_{m}=(i, j)$, construct two ADG section assignment nodes that express:

$$
\begin{aligned}
& A_{m}(:, 1)=t_{i} \\
& A_{m}(1,:)=t_{j} .
\end{aligned}
$$

Claim: For any $k \leq|V|$, the ADG has an axis assignment with $k$ template dimensions iff graph $G$ is $k$-colorable.

Let each color correspond to a template dimension, and $k$ be the dimensionality of the template. Each array $t_{i}$ is assigned to dimension $f\left(v_{i}\right)$ in the template, and each array $A_{m}$ spans two dimensions in the template: the dimensions $f\left(v_{i}\right)$ and $f\left(v_{j}\right)$.

(IF) Assume that $G$ is $k$-colorable, then the mapping described above yields an assignment of template dimensions to array axes that does not violate the constraint that the axes of a given object must lie in different template dimensions.

(ONLY IF) Assume that the ADG has a legal assignment of axes to template dimensions and the mapping described above is constructed. Then the assignment does not violate the constraints of the colorability problem that the endpoints of each edge must have different colors.

In practice, however, the axis quotient graph is usually easy to color and standard heuristics [2] find an optimal coloring.

\section{Contracting the Constraint Graph}

The constraint graph may be contracted into a smaller graph that captures all of the alignment constraints and costs of the original graph. We can then use the algorithm of Section 3, or any other method, to align the contracted graph, and propagate the results back to the original graph by reversing the contractions. For many examples, the contracted constraint graph has only a few vertices. Since performing the contractions is inexpensive compared to doing the alignment, contraction makes the total running time much smaller.

\subsection{Contraction Operations}

The contractions rely on the following property of D-matrices.

Lemma 2 Let $Y$ and $C$ be given D-matrices. There is always at least one D-matrix $X$ such that $X C=Y$. Proof: Let $X$ be a $(p \times q)$ matrix, $C$ be $(q \times r)$, and $Y$ be $(p \times r)$. Without loss of generality, the rows and columns of the matrices may be permuted to place $C$ in upper diagonal form. Now, if $q=r$, then $C$ is a diagonal matrix and $X$ is uniquely determined. Otherwise, $q>r$ and the problem may be written as follows:

$$
Y=\left[X_{1} X_{2}\right]\left[\begin{array}{c}
D \\
0
\end{array}\right] .
$$

$D$ is a nonsingular $(r \times r)$ matrix, $X_{1}$ is $(p \times r)$ and $X_{2}$ is $(p \times(q-r))$. Matrix $X_{1}$ is fully specified by $Y$ and $D$. Any value for $X_{2}$ satisfies the equation, but it must be chosen so that $X$ is a D-matrix, which is simple. The $r$ columns of $X_{1}$ are multiples of $r$ standard basis vectors $\left\{e_{1}, e_{2}, \cdots, e_{p}\right\}$. The $(q-r)$ columns of $X_{2}$ must be multiples of the remainder and there are $(p-r)$ such columns remaining. Since $(p \geq q)$, there is at least one way in which this can be achieved.

We now present four situations where the $\mathrm{CG}$ can be contracted. 
Contraction 1: Suppose vertex $v$ has degree 1, so $v$ is adjacent to only one other vertex $w$. The edge between them has a directed constraint $C_{v w}$ or $C_{w v}$. In either case, contract the graph $G$ into a smaller graph $G^{\prime}$ by removing $v$ and the edge. To convert an alignment for $G^{\prime}$ into one for $G$ with the same cost, choose a position $P_{v}$ for $v$ as follows. If the edge was directed $(w, v)$, then compute $P_{v}=P_{w} C_{w v}$. If the edge was directed $(v, w)$, solve $P_{v} C_{v w}=P_{w}$ for $P_{v}$ by Lemma 2 .

Contraction 2: The second contraction applies when a vertex $v$ is adjacent to only two different vertices. In this case there is an edge $(u, v)$ and an edge $(v, w)$, and $u \neq v \neq w \neq u$. Construct $G^{\prime}$ by eliminating $v$ and contracting the two edges into a new edge $(u, w)$ with edge label $C_{u w}=C_{u v} C_{v w}$, and weight $W_{u w}=\min \left(W_{u v}, W_{v w}\right)$. To convert an alignment for $G^{\prime}$ into one for $G$ with the same cost, choose a position for $v$ as follows.

There are two cases. If the alignment for $G^{\prime}$ satisfies edge $(u, w)$, then compute $P_{v}=P_{u} C_{u v}$. This satisfies $(u, v)$ in $G$, and $(v, w)$ is also satisfied because $P_{w}=P_{u} C_{u w}=P_{u} C_{u v} C_{v w}=P_{v} C_{v w}$. If the alignment for $G^{\prime}$ does not satisfy $(u, w)$, then $(u, w)$ contributes cost $W_{u w}=\min \left(W_{u v}, W_{v w}\right)$ to $G^{\prime}$. We can construct an alignment for $G$ with the same cost by failing to satisfy the less expensive of $(u, v)$ and $(u, w)$ : If $W_{v w}<W_{u v}$ then let $P_{v}=P_{u} C_{u v}$, and if $W_{u v} \leq W_{v w}$ the solve $P_{w}=P_{v} C_{v w}$ for $P_{v}$ by Lemma 2 .

Contractions 3 and 4: There are two final contraction operations. Merge parallel edges if their constraint matrices are equal and add their edge weights. Finally, reverse edges with invertible constraints. (Note that all square D-matrices are invertible.) This may enable other contraction operations.

Figure 4 illustrates these contraction operations. The initial constraint graph corresponds to the example program and ADG of Figure 1. Contraction eliminates dangling acyclic branches and combines edges in series. The result is a very simple graph capturing the essential structure of the problem.

\section{Experimental Results}

To illustrate our algorithm, we constructed the two small example programs shown in Figure 5, which have nontrivial axis alignment issues.

We generated alignments for the programs using our algorithms with various edge selection rules and ran the optimized programs on the CM-5 to measure the effect of alignment on their running times. Because the CMF compiler does not allow axis-changing alignments [11], we broke alignment into two parts. We performed axis alignment manually by changing the orientation of the arrays in the program and including explicit array transpose operations for unsatisfied CG edges. We specified stride alignment by adding ALIGN directives to the source code.

We did three kinds of experiments. First, we examined the effect of edge selection strategy on the quality of solutions found. Second, we examined the effect of axis and stride alignment on running time, and the correlation between the discrete metric of the optimization problem and the actual running time on 


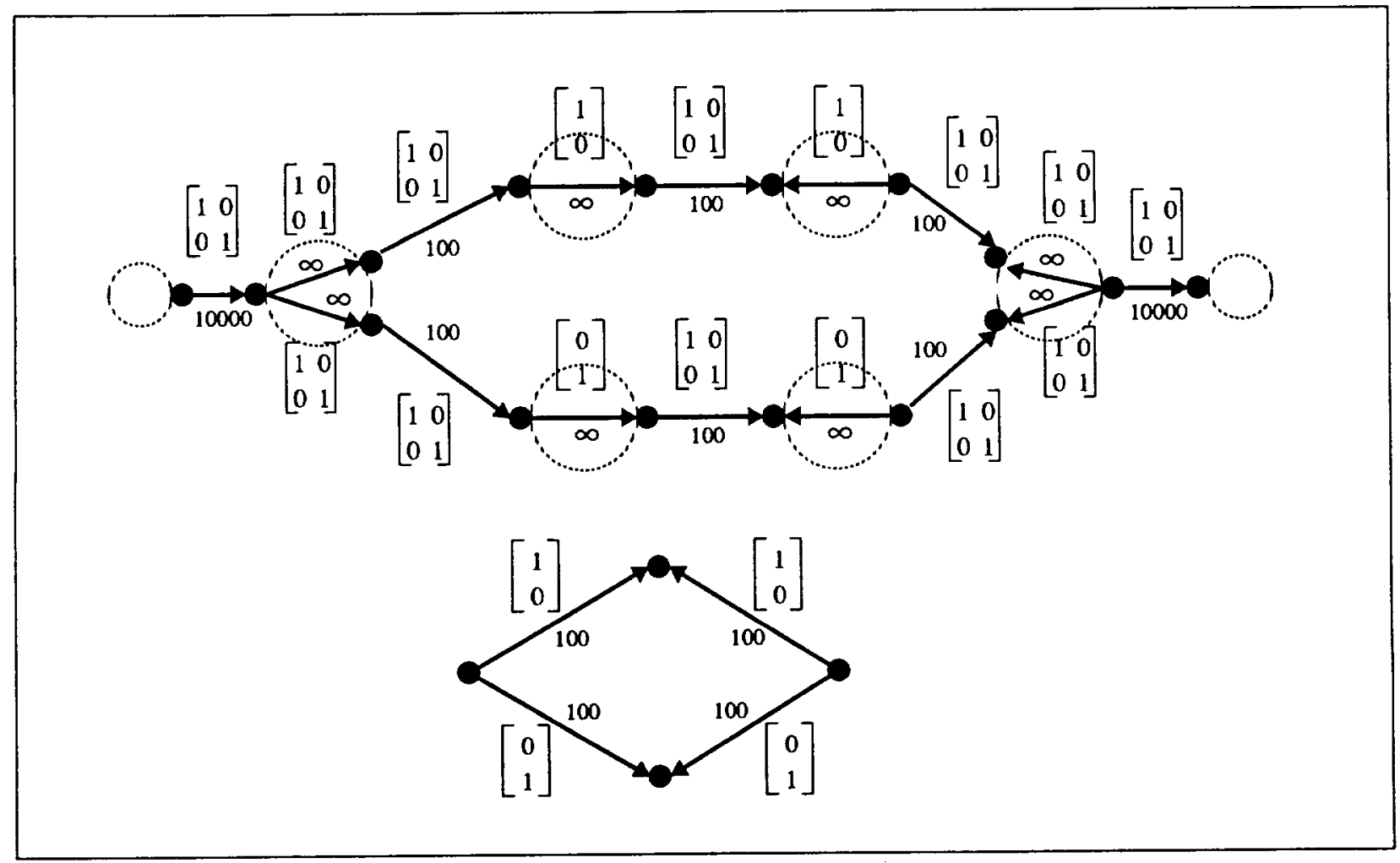

Figure 4: The constraint graph corresponding to the ADG of Figure 1 and a contracted form. In this example, the contraction operations remove most of the extraneous information from the graph leaving only its essential structure. 


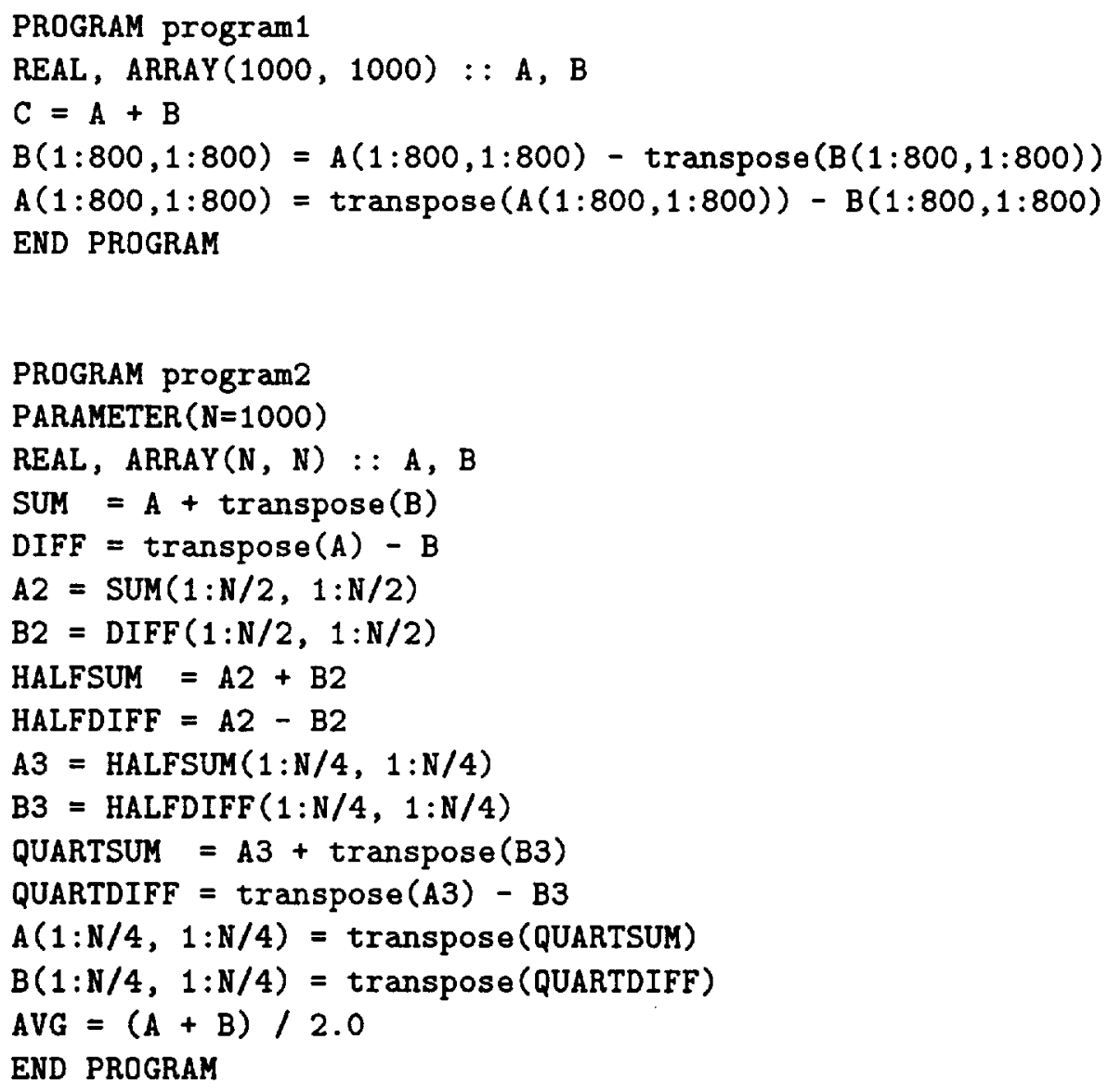

Figure 5: Two example programs. 
Table 1: The estimated and actual times of two programs under differing axis and stride alignments.

\begin{tabular}{|l|l|r|r|}
\hline Example & Method & $\begin{array}{r}\text { Communication } \\
\text { Cost }\end{array}$ & $\begin{array}{r}\text { CM5 running } \\
\text { time (secs) }\end{array}$ \\
\hline \hline PROGRAM1 & (none) & 1280000 & .25 \\
& max-wt & 1000000 & .25 \\
& min-wt & 1280000 & .13 \\
& random & 1000000 & .25 \\
& (optimal) & & .13 \\
\hline PROGRAM2 & (none) & 1750000 & .56 \\
& max-wt & 1375000 & .40 \\
& min-wt & 1312500 & .34 \\
& random & 1312500 & .34 \\
\hline
\end{tabular}

the CM-5. Third, we examined the effect of graph contraction on the time required to find a solution.

\subsection{Edge Selection Ordering}

At each iteration the optimization algorithm removes an edge from the bag. We examined three edge selection strategies: maximum weight first, minimum weight first and random selection. With the maximum weight ordering, our algorithm reduces to the greedy heuristic proposed by Knobe, Lukas and Steele. However, our experimental results show that other orderings (combined with the min-cut procedure) can yield superior results.

The contracted graph for PROGRAM1 appears in Figure 6 with two different alignments. One requires communication on two edges, for a total cost of $1,280,000$. The optimal solution requires communication on only one edge and costs $1,000,000$. This optimal solution was found using the minimum edge-weight heuristic and the min-cut procedure. This example demonstrates a shortcoming of the maximum-weight heuristic: the optimal solution in this example cannot be found if edges are added in order of decreasing weight, because the subgraph of heaviest edges is satisfiable, but not optimal.

\subsection{Execution Time}

We measured the execution time of each of the programs on the CM-5 with each alignment our algorithm generated, and also without axis or stride optimization as a baseline. The times measured were averaged over ten runs. Table 1 presents the data. The solution reported for the random edge selection heuristic reflects the best of five trials. The table shows the estimated cost according to the discrete metric and the actual execution time of the program.

We draw two conclusions. First, optimizing axis and stride alignment can significantly improve the running time of the programs. Second, our discrete metric of communication cost is an accurate enough measure to correctly predict the relative running times with different alignments. 


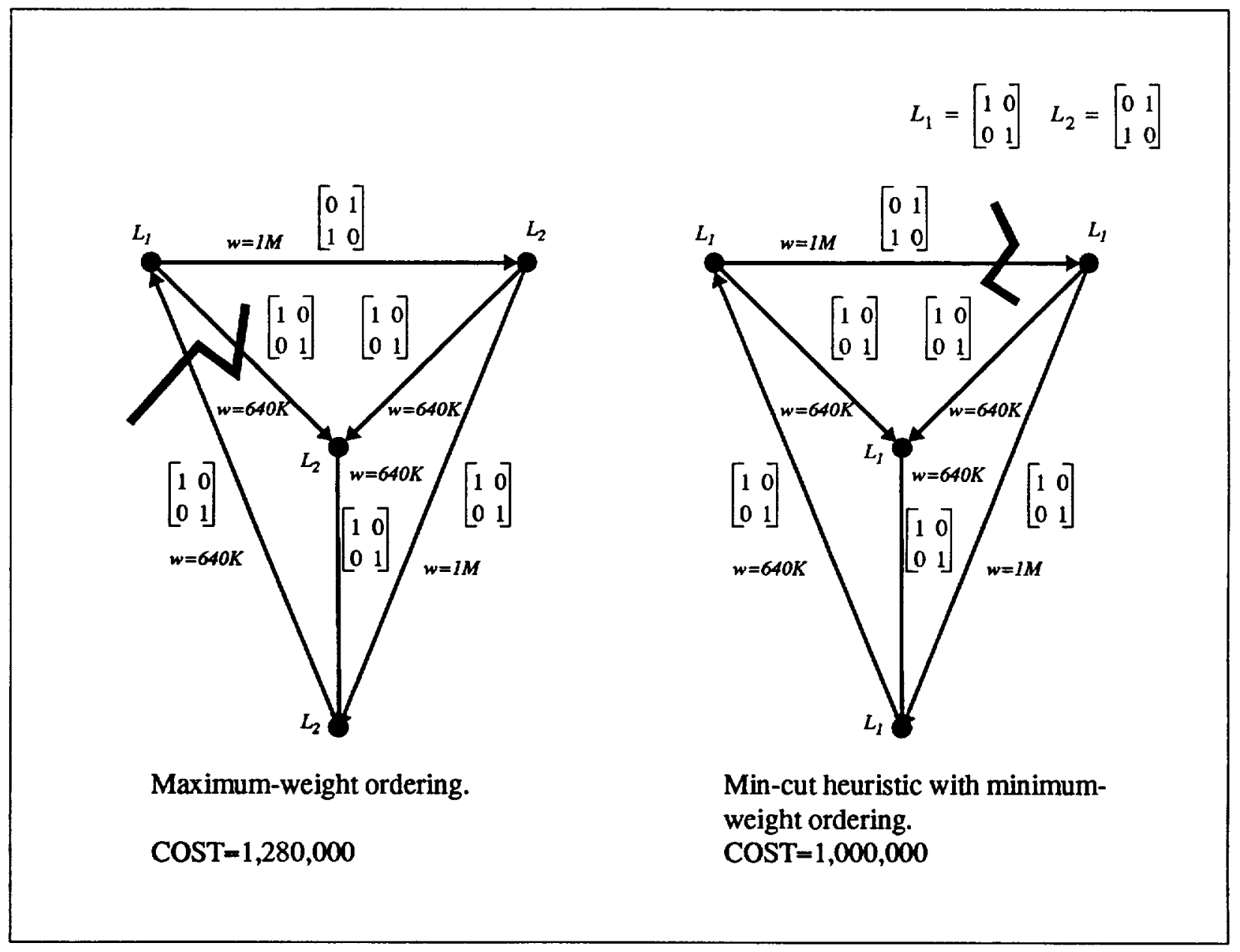

Figure 6: The contracted graph for PROGRAM1. Alignments are shown by a "cut" through the unsatisfied edges. The optimal alignment requires cutting one of the heaviest edges. The maximum-weight heuristic cannot find this alignment. 
Table 2: The effect of contraction on the quality of solutions produced and the running time of the algorithm.

\begin{tabular}{|c|c|c|c|c|}
\hline \multirow[b]{2}{*}{$\begin{array}{l}\text { Example/ } \\
\text { Method }\end{array}$} & \multicolumn{2}{|c|}{ FULL GRAPH } & \multicolumn{2}{|c|}{ CONTRACTED } \\
\hline & $\begin{array}{r}\text { Communication } \\
\text { Cost }\end{array}$ & $\begin{array}{r}\text { Optimization } \\
\text { Time (secs) }\end{array}$ & $\begin{array}{r}\text { Communication } \\
\text { Cost }\end{array}$ & $\begin{array}{r}\text { Optimization } \\
\text { Time (secs) }\end{array}$ \\
\hline$\overline{\text { PROGRAM1 }}$ & \multicolumn{2}{|c|}{ (44 nodes, 47 edges) } & \multicolumn{2}{|c|}{ (4 nodes, 6 edges) } \\
\hline $\max -w t$ & 1280000 & .95 & 1280000 & .23 \\
\hline $\min -w t$ & 1000000 & 1.50 & 1000000 & .23 \\
\hline random-best & 1280000 & 2.22 & 1280000 & .19 \\
\hline random-worst & 1920000 & 1.72 & 1640000 & .25 \\
\hline PROGRAM2 & \multicolumn{2}{|c|}{ (70 nodes, 77 edges) } & \multicolumn{2}{|c|}{ (14 nodes, 21 edges) } \\
\hline $\max -w t$ & 1678500 & 2.47 & 1750000 & .40 \\
\hline $\min -w t$ & 2500000 & 6.12 & 1375000 & .58 \\
\hline random-best & 1312500 & 7.14 & 1312500 & .63 \\
\hline random-worst & 2375000 & 4.93 & 2625000 & .65 \\
\hline
\end{tabular}

\subsection{Graph Contraction}

Graph contraction, which has not been suggested elsewhere for this problem, significantly reduces the size of the problem and the solution space that must be examined. Using contraction leads to a large decrease in the running time of the algorithm. In many cases the resulting graphs are small enough that their alignment problems could even be solved exactly by an exhaustive search.

Table 2 shows the running time of the optimization algorithm and the quality of solutions produced with and without contraction. The table reports running times for the entire optimization program, including contraction if any, on a Sun-4/370. Graph contraction is an inexpensive operation, and the time spent reducing the size of the graph is easily recovered by the time saved in the optimization phase.

Although contraction preserves the cost of the optimal alignment, it can change the result of our algorithm because the heuristic is sensitive to the order of selection of equal-weight edges. When optimizing PROGRAM2 with the maximum-weight ordering, a slightly worse solution is found with contraction enabled. In some cases, the contraction phase had the unfortunate effect of reordering the edges so that a worse solution is found.

We explored the effect that contraction has on the quality of solutions found, initially suspecting that contraction leads to better solutions. However, this is not necessarily the case. Figure 7 shows a histogram of the frequency with which alignments of different costs were found by running the algorithm 1000 times with the random edge selection rule. Black bars are alignment costs found when using contraction, and gray bars are costs found without contraction. Using the random edge selection rule, contraction had little effect on the distribution of results.

In all cases, contraction significantly decreases the running time required by the algorithm. This makes it possible to run the optimization algorithm with a number of edge ordering heuristics and then choose the best solution found. 

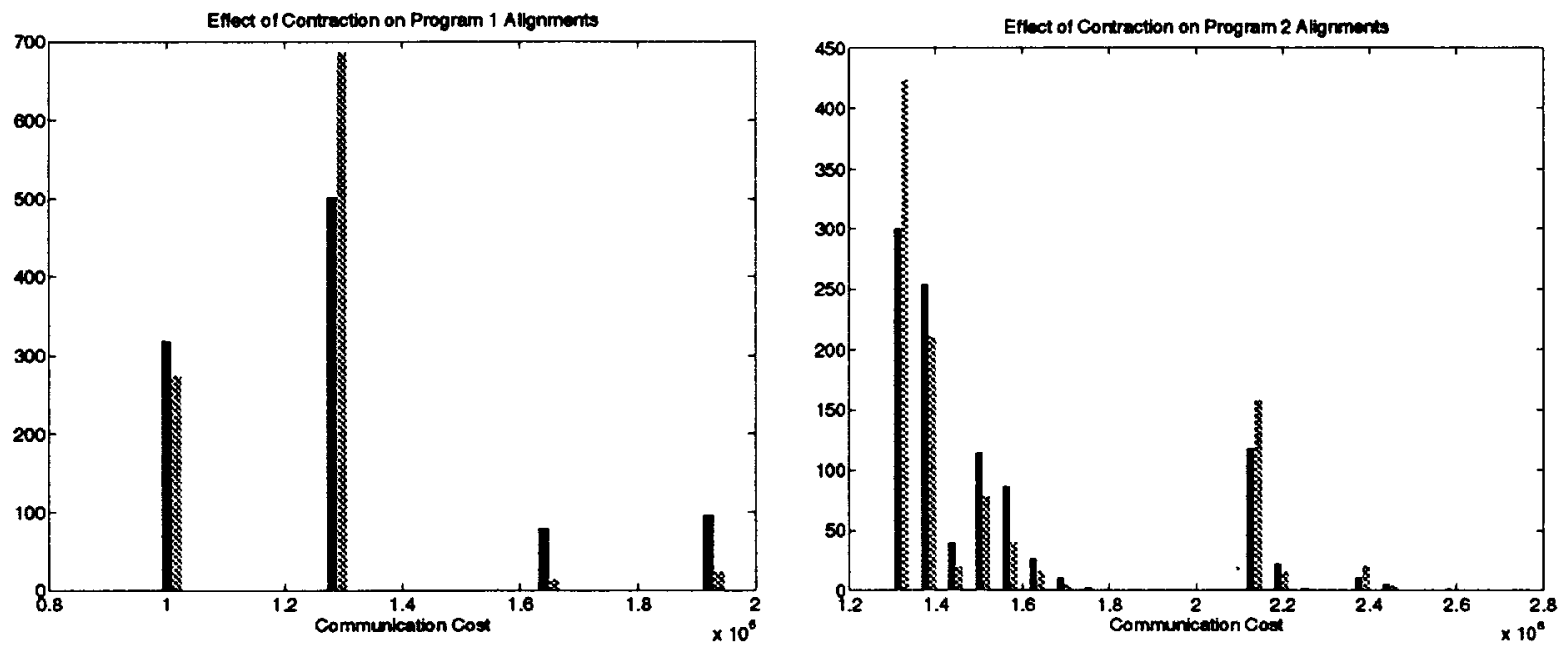

Figure 7: The effect of contraction on the quality of solutions found over 1000 runs of the algorithm using the random edge selection rule. Black bars show alignment costs found when using contraction; gray bars show costs found without contraction. Surprisingly, the distribution of results found is unchanged when contraction is used.

\section{Conclusions}

This paper presents a new approach to axis and stride alignment to optimize array placement in data-parallel programs. Our algorithms extend those previously reported in a number of ways.

Our algorithms use a problem formulation based on the ADG representation. The ADG makes explicit all array objects generated by a program-named arrays as well as unnamed temporaries. Thus, the optimization algorithm has complete control over the placement of every array generated. The ADG also incorporates the effects of control flow into its data flow representation; this information can affect alignment decisions. Other work has not treated control flow as completely.

The graph contraction operations greatly reduce the computation time of the program. For many examples, the contracted constraint graph becomes a graph of only a few vertices, and the alignment problem can be solved exactly. Even when an exact method is not feasible, the reduced size of the contracted graph makes possible a more complete search of the space of possible solutions. We believe that even more powerful graph contractions are possible; indeed we hope eventually to define a set of contractions that reduces most programs enough that optimal alignments can be found by an exponential search procedure.

Axis and stride alignment is a discrete optimization problem. The optimization algorithm we propose is actually a family of optimization algorithms parameterized by an edge ordering. For one particular ordering, our algorithm reduces to the algorithm of Knobe, Lukas and Steele. However, we have shown that other orders, coupled with the min-cut procedure, can lead to superior solutions. Edge orderings based on graph structure may be possible, and there may be more efficient means of finding conflicting edge sets. We intend to investigate these issues in the future. 


\section{Acknowledgements}

David Bau and Lenny Oliker implemented an earlier version of the axis, stride, and offset alignment algorithms, and our experiments here are built on their testbed. The proof of Theorem 1 is also due to David Bau. We thank both of them as well as Jingke Li and Shang-Hua Teng for stimulating discussions of all these problems.

\section{References}

[1] Jennifer M. Anderson and Monica S. Lam. Global optimizations for parallelism and locality on scalable parallel machines. In Proceedings of the ACM SIGPLAN'93 Conference on Programming Language Design and Implementation, pages 112-125, Albuquerque, NM, June 1993.

[2] Gregory J. Chaitin, Marc A. Auslander, Ashok K. Chandra, John Cocke, Martin E. Hopkins, and Peter W. Markstein. Register allocation via coloring. Computer Languages, 6:47-57, 1981.

[3] Siddhartha Chatterjee, John R. Gilbert, and Robert Schreiber. Mobile and replicated alignment of arrays in data-parallel programs. In Proceedings of Supercomputing'93, pages 420-429, Portland, OR, November 1993. Also available as RIACS Technical Report 93.08 and Xerox PARC Technical Report CSL-93-7.

[4] Siddhartha Chatterjee, John R. Gilbert, Robert Schreiber, and Thomas J. Sheffler. Modeling dataparallel programs with the alignment-distribution graph. Journal of Programming Languages, 1994. Special issue on compiling and run-time issues for distributed address space machines. To appear.

[5] Ron Cytron, Jeanne Ferrante, Barry K. Rosen, Mark N. Wegman, and F. Kenneth Zadeck. Efficiently computing static single assignment form and the control dependence graph. ACM Transactions on Programming Languages and Systems, 13(4):451-490, October 1991.

[6] Michael R. Garey and David S. Johnson. Computers and Intractability: A Guide to the Theory of NP-Completeness. W. H. Freeman and Company, San Francisco, CA, 1979.

[7] High Performance Fortran Forum. High Performance Fortran language specification. Scientific Programming, 2(1-2):1-170, 1993.

[8] Kathleen Knobe, Joan D. Lukas, and William J. Dally. Dynamic alignment on distributed memory systems. In Proceedings of the Third Workshop on Compilers for Parallel Computers, pages 394-404, Vienna, Austria, July 1992. Austrian Center for Parallel Computation.

[9] Kathleen Knobe, Joan D. Lukas, and Guy L. Steele Jr. Data optimization: Allocation of arrays to reduce communication on SIMD machines. Journal of Parallel and Distributed Computing, 8(2):102-118, February 1990.

[10] Jingke Li and Marina Chen. The data alignment phase in compiling programs for distributed-memory machines. Journal of Parallel and Distributed Computing, 13(2):213-221, October 1991.

[11] Thinking Machines Corp. CM Fortran User's Guide for the CM-5, version 2.1 edition, January 1994. 
[12] Skef Wholey. Automatic Data Mapping for Distributed-Memory Parallel Computers. PhD thesis, School of Computer Science, Carnegie Mellon University, Pittsburgh, PA, May 1991. Available as Technical Report CMU-CS-91-121. 